\title{
Liquid biopsy: Novel perspectives on the importance and spectrum of PIK3CA, PTEN and RET mutations in solid tumors
}

\author{
IBRAHIM SAHIN $^{1}$, HANIFE SAAT $^{1}$, SERCAN AKSOY $^{2}$, OMER DIZDAR $^{2}$, \\ HAKTAN BAGIS ERDEM ${ }^{3}$ and TAHA BAHSI ${ }^{3}$
}

\author{
${ }^{1}$ Department of Medical Genetics, University of Health Sciences, Dışkapı Yıldırım Beyazıt Training and Research Hospital, \\ 06110 Ankara; ${ }^{2}$ Department of Medical Oncology, Hacettepe University Cancer Institute, 06590 Ankara; \\ ${ }^{3}$ Department of Medical Genetics, University of Health Sciences, \\ Dr. Abdurrahman Yurtaslan Ankara Oncology Training and Research Hospital, 06105 Ankara, Turkey
}

Received May 1,2021; Accepted July 28, 2021

DOI: $10.3892 / \mathrm{mco} .2021 .2434$

\begin{abstract}
Many people die from lung and breast cancer. Consequently, both physicians and researchers strive to provide reliable monitoring for disease, diagnosis and prognosis as well as resistance prediction. In the present study, a comprehensive liquid biopsy panel was performed on 474 patients to examine the importance and spectrum of recurrent somatic cancer mutations. Most patients visited the clinic with a diagnosis of advanced resistant cancer. The patients underwent a comprehensive liquid biopsy panel. Patients were divided into four groups based on cancer type as follows: Lung ( $\mathrm{n}=379,79.9 \%)$, breast $(\mathrm{n}=72,15.2 \%)$, gastrointestinal $(n=11,2.3 \%)$ and other $(n=12,2.5 \%)$. Tier I-II-III classified variants were included in the study. The mean age was 60 years, with a range of 20-86 years. There were notably more male $(\mathrm{n}=272,57.4 \%)$ than female patients $(\mathrm{n}=202,42.6 \%)$. The most commonly mutated genes were TP53, EGFR, PIK3CA, RET, PTEN, MET, ATM and KRAS. The most common mutations were 'PIK3CA, c.3140A $>$ G, p.His1047Arg', 'RET, c.2324delinsGAC, p.Glu775Glyfs*6', 'TP53, c. $217 \mathrm{G}>\mathrm{C}$, p.Val73Leu', 'EGFR, c.2155G $>$ A, p.Gly719Ser', 'PIK3CA, c.1624G>A, p.Glu542Lys', 'PTEN, c.397G >A, p.Val133Ile' and 'EGFR, c.2235_2249del, p.Glu746_ Ala750del'. The PIK3CA, PTEN and RET variants showed a higher incidence in the breast and lung groups compared with other groups. To the best of our knowledge, the present study is the first to concentrate on PIK3CA, PTEN and RET mutations in the context of breast and lung adenocarcinoma and to evaluate both genetic variability and the effect of treatment. The present results showed that patients with
\end{abstract}

Correspondence to: Dr Ibrahim Sahin, Department of Medical Genetics, University of Health Sciences, Dışkapı Yıldırım Beyazıt Training and Research Hospital, 20 Ziraat Mah. Şehit Ömer Halisdemir Cad, 06110 Ankara, Turkey

E-mail: ibrahimsahinmd@gmail.com

Key words: liquid biopsy, PIK3CA, PTEN, RET solid tumors, particularly lung and breast cancer, may benefit from PIK3CA, PTEN and RET sequencing to assess clinical characteristics and prognosis. Discoveries regarding the gene structure and mechanisms of PIK3CA, PTEN and RET may inform more clinically meaningful therapeutic approaches for patients with cancer and serve an essential role in improving individual risk prediction, therapy and prognosis.

\section{Introduction}

More people die of cancer than any other disease in the world today (1). Accordingly, both physicians and researchers strive to provide reliable monitoring for disease, diagnosis and prognosis as well as resistance prediction. The primary goal is to provide patients with adequate treatment and restore their well-being. Lung cancer is the most common malignancy and contributes to the greatest number of cancer deaths (2). Colorectal cancer is the third-most frequently contracted malignant disease worldwide (3). Among women, breast cancer is the most common type of cancer (4).

In previous years, liquid biopsy techniques have been used to treat a number of different types of cancer (5-7). This less invasive testing method (compared with traditional biopsy) offers potential for a satisfactory outcome, higher recovery rate and more accurate results $(5,6)$. Changes in circulating tumor DNA (ctDNA) are used for cancer screening in asymptomatic people, detecting mutations for theranostic consideration and monitoring tumor dynamics and genetic evolution (5). Repeated analysis and quantitation of ctDNA may provide information on changes in clonal composition over time, allowing for modification of treatment regime (8). KRAS, BRAF and $E G F R$ mutations may be identified via liquid biopsy in patients with colon cancer, melanoma and lung cancer $(5,6)$.

Numerous types of tumor are dependent on oncogenes: Oncogene addiction has been noted in numerous types of neoplasm, such as lung cancer (9). Among patients with lung adenocarcinoma, $\sim 50 \%$ have at least one driver mutation representing a potential target for clinical intervention. For example, activating EGFR mutations are predictive of susceptibility to $E G F R$ inhibitors, such as erlotinib and gefitinib. ctDNA analysis has been shown to diagnose EGFR exon 19 
deletions and L858R mutations with a sensitivity of $82-87 \%$ and a precision of $97-98 \%$ and may thus be an alternative to tissue genetic analysis. Most patients taking EGFR-inhibiting medication experience disease development after 2 years of therapy and $60 \%$ of resistance to treatment is due to a disease clone containing a secondary EGFR T790M mutation, which inhibits drug access to the target kinase. T790M is more common in exon 19 deletion than in L858R among patients with acquired resistance to EGFR tyrosine kinase inhibitors (TKIs) $(7,10)$.

Among patients with non-small cell lung cancer (NSCLC), $1 \%$ have chromosome translocations in the RET gene. The College of American Pathologists, the International Association for the Study of Lung Cancer and the Association for Molecular Pathology published recommendations including ROS1 testing for all patients with adenocarcinoma, the use of additional genes (ERBB2, MET, BRAF, KRAS and RET) for laboratories performing next-generation sequencing (NGS) panels and immunohistochemistry as an alternative to fluorescence in situ hybridization. Acquired resistance mutation C797S may occur in tumors that have progressed following osimertinib treatment with T790M mutation (7).

It is estimated that $30-40 \%$ of estrogen and/or progesterone receptor-positive breast cancer cases have PIK3CA mutations (11). Increased activation of the PI3K/AKT/mTOR pathway contributes to various aspects of cancer, including acquired growth signals, inhibition of apoptosis, vessel generation and insensitivity to anti-growth signals. The $\mathrm{PI} 3 \mathrm{~K} / \mathrm{AKT} / \mathrm{mTOR}$ pathway is associated tumor development and progression in lung cancer. Therefore, this pathway represents a novel target for anticancer treatment (12). The most validated anti-oncogenic effect of PTEN is inhibition of the PI3K/AKT/mTOR oncogenic signaling system; other documented effects include chromosomal integrity and DNA repair (13).

Cancer-associated gene variants may be found in apparently healthy people, arising in part from clonal hematopoiesis (14). Age-associated clonal hematopoiesis, often referred to as indeterminate potential clonal hematopoiesis, is distinguished by recurrent somatic variants that are associated with peripheral blood hematological cancer. The most commonly involved genes are DNMT3A, TET2 and ASXL1; other genes that are often mutated include TP53, JAK2, SF3B1, GNB1, PPM1D, GNAS and BCORL1. Due to limited data, caution is required when interpreting ctDNA variants in these genes and more research is needed to understand how to interpret and report ctDNA variants in these genes $(14,15)$.

In the present study, a comprehensive liquid biopsy panel was performed on 474 patients to assess the importance and spectrum of recurrent cancer somatic mutations.

\section{Materials and methods}

Patients. The present study was approved by the Ethics Committee at the University of Health Sciences, Dr. Abdurrahman Yurtaslan Ankara Oncology Training and Research Hospital (Ankara, Turkey). Written informed consent was obtained from all patients. Most patients visited the Medical Genetics Clinic at the Department of Medical Genetics, University of Health Sciences, Dışkapı
Yıldırım Beyazıt Training and Research Hospital and the Department of Medical Genetics, University of Health Sciences, Dr. Abdurrahman Yurtaslan Ankara Oncology Training and Research Hospital (Ankara, Turkey) with a diagnosis of advanced resistant cancer. Clinical histories and molecular results were reviewed for 474 patients examined at the Department of Medical Oncology, Hacettepe University, Faculty of Medicine, Department of Medical Genetics, University of Health Sciences, Dışkapı Yıldırım Beyazit Training and Research Hospital and the Department of Medical Genetics, University of Health Sciences, Dr. Abdurrahman Yurtaslan Ankara Oncology Training and Research Hospital (Ankara, Turkey). The patients underwent a comprehensive liquid biopsy panel between January 2018 and December 2020 at the Ankara Central Genetic Laboratory (Turkey). They were evaluated according to the National Comprehensive Cancer Network guidelines for breast-ovarian cancer and Lynch syndrome $(16,17)$. Patients with a strong family history of cancer underwent a familial cancer panel. Patients with missing data were excluded.

Patients were divided based on cancer type into the following groups: Lung $(n=379,79.9 \%)$, breast $(n=72,15.2 \%)$, gastrointestinal $(n=11,2.3 \%)$ and other $(n=12,2.5 \%)$. The other group included patients with rare or unspecific cancer (including carcinoma of unknown primary, melanoma and bladder, gallbladder, liver, laryngeal and endometrial cancer).

DNA panels and NGS. From blood samples $(10 \mathrm{ml})$ collected in EDTA tubes, genomic DNA was extracted according to the manufacturer's procedure using a QIAamp DNA Blood Midi kit and QIAcube (both Qiagen, Inc.). Paired-end sequencing was performed with a loading concentration of $1.6 \mathrm{pM}$. The concentration was measured with an Invitrogen Qubit 3 Fluorometer (Thermo Fisher Scientific, Inc.). Amplicon lengths were 265 bp for Sophia Genetics 56 G Oncology Solution (Sophia Genetics) and 250 bp for the ArcherDx Reveal ctDNA 28 kit (ArcherDx, Inc.).

Two different multigene panels were used: ArcherDx Reveal ctDNA 28 kit (AKT1, CTNNB1, ESR1,IDH2, MAP2K2, NTRK1, RET, ALK, DDR2, FGFR1, KIT, MET, NTRK3, ROS1, AR, EGFR, HRAS, KRAS, MTOR, PDGFRA, SMAD4, $B R A F, E R B B 2, I D H 1, M A P 2 K 1, N R A S, P I K 3 C A, T P 53)$ and Sophia Genetics $56 \mathrm{G}$ Oncology Solution (ABL1, AKT1, ALK, $A P C, A T M, B R A F, C D H 1, C D K N 2 A, C S F-1 R, C T N N B 1$, DDR2, DNMT3A, EGFR, ERBB2, ERBB4, EZH2, FBXW7, FGFR1, FGFR2, FGFR3, FLT3, FOXL2, GNA11, GNAQ, GNAS, HNF1A, HRAS, IDH1, IDH2, JAK2, JAK3, KDR, KIT, KRAS, MAP2K1, MET, MLH1, MPL, MSH6, NOTCH1, NPM1, NRAS, PDGFRA, PIK3CA, PTEN, PTPN11, RB1, RET, STK11, SMAD4, SMARCB1, SMO, SRC, TP53, TSC1, VHL). The Sophia Genetics 56G Oncology Solution was used between January 2018 and November 2020 and ArcherDx Reveal ctDNA 28 kit has been used since January 2020. The sequencing was performed on an Illumina MiSeq system (Illumina, Inc.). The data were analyzed using the Archer Analysis Platform (ArcherDx, Inc.) for the ArcherDx Reveal ctDNA 28 kit and Sophia DDM software v4 (Sophia Genetics) for the Sophia Genetics 56G Oncology Solution. Visualization of the data was performed with Integrative Genomics Viewer 2.7.2 (Broad Institute) software. 

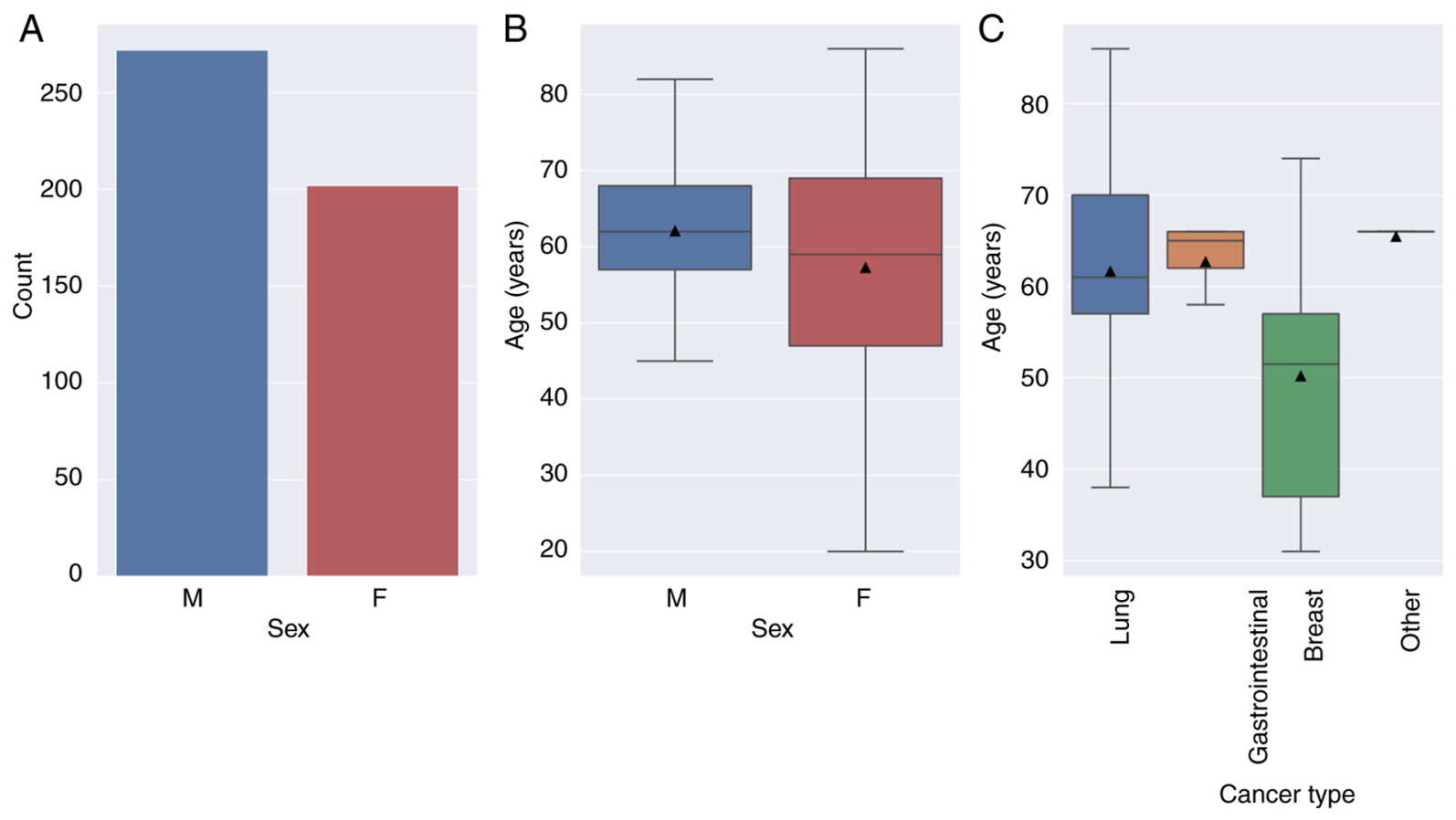

Figure 1. Patient characteristics and groups. (A) Sex distribution of patients. Mean (black triangle) and median (black line) age of patients separated by (B) sex and (C) cancer type. $\mathrm{M}$, male; F, female.

Statistical analysis. The available evidence (population frequency information, case notes, case/control and functional tests, internal co-occurrence and co-segregation data, evolutionary conservation data and in silico predictions) for all variants, except previously characterized benign alterations, was thoroughly evaluated and analyzed. Data are presented as the mean. In compliance with the recommendations issued by the Association for Molecular Pathology, the American Society of Clinical Oncology and the College of American Pathologists, variants were categorized into tiers as follows: I, variants with strong clinical significance; II, variants with potential clinical significance; III, variants with unknown clinical significance and IV, variants that are benign or likely benign (18). Tier I, II and III variations were included in the study. Figs. 1-3 were prepared using Python (version 3.9.2, https://docs.python.org); Fig. 4 was prepared using Lollipops-v1.3.5 (19).

\section{Results}

Patients. The mean age of the participants was 60 years, with a range of 20-86 years (Fig. 1). Most patients were between the ages of 50 and 70 years. The mean age in each group was as follows: breast, 50.2; gastrointestinal, 62.7; lung, 61.6 years and other, 65.5 years. Patients with advanced resistant breast cancer were referred to the clinic at an earlier age when compared with other groups. There were notably more male patients $(n=272,57.4 \%)$ than females $(n=202,42.6 \%$; Fig. 1).

Mutations. Mutations were detected in 357 patients and the majority of variant fractions were $0.1-10.0 \%$ (data not shown). A total of 131 mutations were nonsense or frameshifts. The most commonly mutated genes detected in patients were TP53, EGFR, PIK3CA, RET, PTEN, MET, ATM and KRAS. The most common mutations detected were 'PIK3CA, c.3140A $>\mathrm{G}$,
p.His1047Arg', 'RET, c.2324delinsGAC, p.Glu775Glyfs*6', 'TP53, c. $217 \mathrm{G}>\mathrm{C}$, p.Val73Leu', 'EGFR, c. $2155 \mathrm{G}>\mathrm{A}$, p.Gly719Ser', 'PIK3CA, c.1624G $>$ A, p.Glu542Lys', 'PTEN, c.397G >A, p.Val133Ile', 'DNMT3A, c.2656C >T, p.Gln886Ter', 'EGFR, c.2235_2249del, p.Glu746_Ala750del', 'SMO, c.1604G >T, p.Trp535Leu' and 'TP53, c.764T >C, p.Ile255Thr'. The 'PIK3CA, c.3140A $>$ G, p.His1047Arg' mutation was observed eight times (2.24\%; Fig. 2). The total number of different $E G F R$ exon 19 deletions exceeded other mutations $(n=9)$.

The most commonly mutated genes in each group were as follows: Breast, TP53 ( $\mathrm{n}=16,23.5 \%)$, PIK3CA $(\mathrm{n}=13,19.1 \%)$, $R E T(\mathrm{n}=6,8.8 \%)$ and $E G F R(\mathrm{n}=4,5.9 \%)$; gastrointestinal, TP53 $(\mathrm{n}=3,42.8 \%), E G F R(\mathrm{n}=2,28.6 \%)$ and $K R A S(\mathrm{n}=2,28.6 \%)$; lung, TP53 ( $\mathrm{n}=59,21.8 \%)$, EGFR $(\mathrm{n}=37,13.7 \%)$, PIK3CA $(\mathrm{n}=18,6.6 \%)$, PTEN $(\mathrm{n}=6,5.9 \%)$, RET $(\mathrm{n}=13,4.8 \%)$, ATM $(\mathrm{n}=11,4 \%)$ and $M E T(\mathrm{n}=11,4 \%)$ and other, TP53 $(\mathrm{n}=4,33.3 \%)$ and $\operatorname{MET}(\mathrm{n}=2,16.6 \%$; Fig. 3$)$.

The most common mutations in each group were as follows: Breast, 'PIK3CA, c.3140A >G, p.His1047Arg', 'RET, c.2324delinsGAC, p.Glu775Glyfs*6' and 'TP53, c.217G>C, p.Val73Leu'; gastrointestinal, 'KRAS, c.194G>A, p.Ser65Asn', 'TP53, c.217G $>$ C, p.Val73Leu' and 'EGFR, c.2155G $>\mathrm{A}$, p.Gly719Ser'; lung, 'PIK3CA, c.1624G $>$ A, p.Glu542Lys', 'EGFR, c.2235_2249del, p.Glu746_Ala750del', 'DNMT3A, c. $2656 \mathrm{C}>\mathrm{T}$, p.Gln886Ter', 'TP53, c.764T >C, p.Ile255Thr', 'RET, c.2324delinsGAC, p.Glu775Glyfs*6', 'EGFR, c.2573T $>$ G, p.L858R', 'TP53, c.524G $>$ A, p.Arg175His', 'RET, c.1784A $>\mathrm{G}$, p.Glu595Gly', 'EGFR, c.2369C $>\mathrm{T}$, p.Thr790Met' and 'PIK3CA, c.3140A $>$ G, p.His1047Arg' and other, 'MET, c.3380T>C, p.Val1127Ala' and 'TP53, c.403T>C, p.Cys135Arg' (Fig. 3).

In 117 (24.7\%) patients, no responsible mutation was identified.TP53,PIK3CA and RET gene mutations were predominant 
A

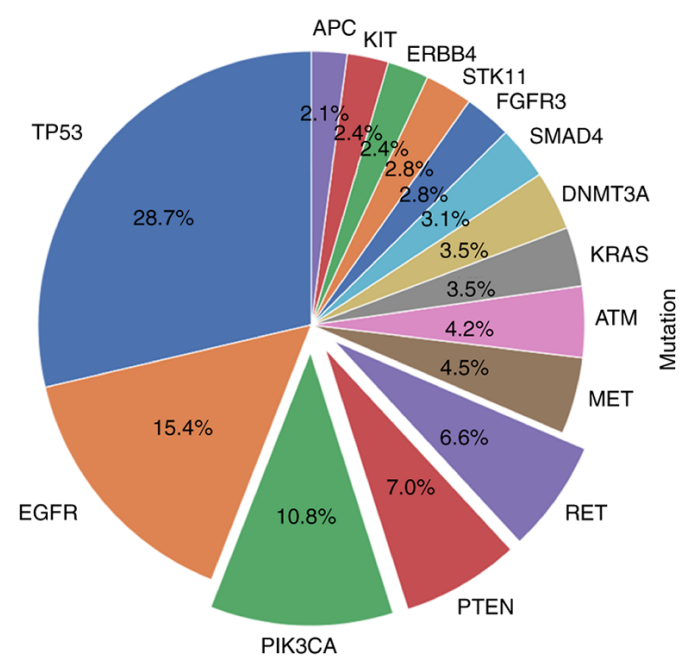

B

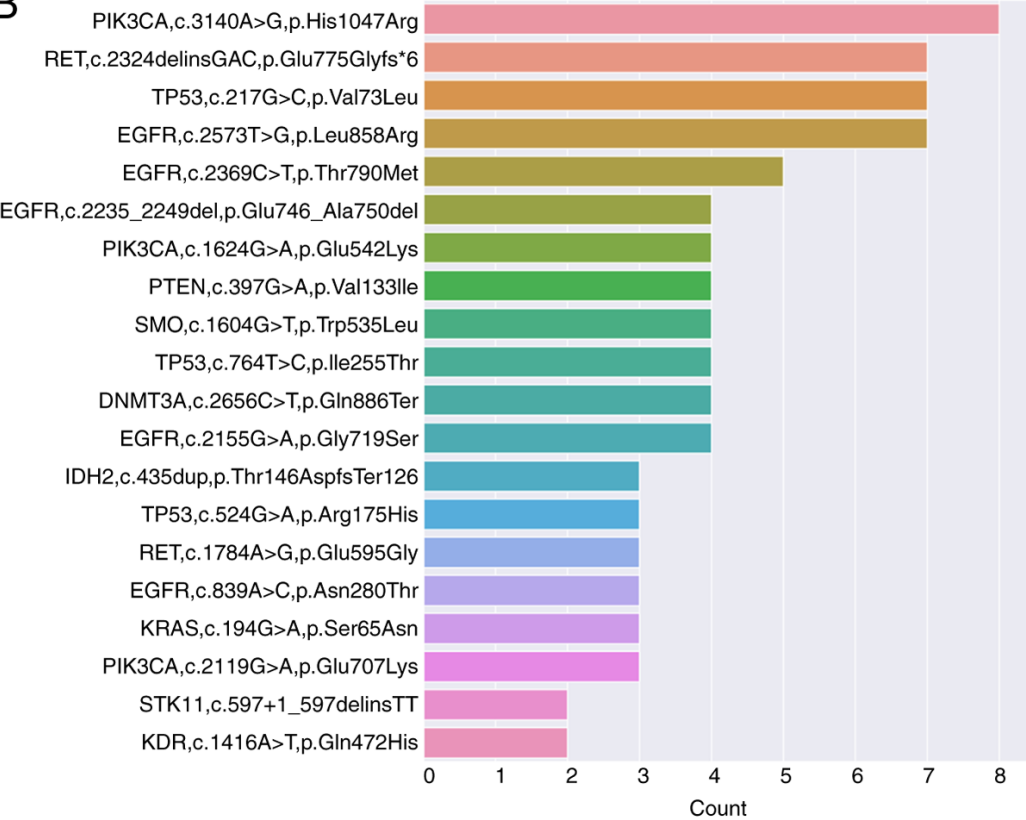

Figure 2. Genes and mutations. (A) Pie chart showing the 15 most commonly mutated genes. (B) Bar chart showing the most common mutations.

in patients aged $<50$ years; in those aged $\geq 50, T P 53, E G F R$, $P I K 3 C A$ and PTEN gene mutations were predominant. PIK3CA, PTEN and RET variants showed a higher incidence in the breast and lung groups (Fig. 3).

\section{Discussion}

TP53, which encodes a tumor suppressor protein, was the most commonly mutated gene. According to the present data, the most commonly mutated genes were TP53, EGFR, PIK3CA, PTEN, RET, MET, ATM, KRAS and DNMT3A. The most common mutation was 'PIK3CA, c.3140A > G, p.His1047Arg.' PIK3CA mutations were primarily detected in domains associated with the PI3K/AKT/mTOR pathway (Fig. 4). Most patients were diagnosed with lung cancer and this distribution varies in comparison with prior research $(11,12)$. 'PIK3CA, c.1624G $>$ A, p.Glu542Lys' and 'PIK3CA, c.1633G $>$ A, p.Glu545Lys' have been reported together, but in the present study these mutations were detected separately and 'PIK3CA, c.1624G $>\mathrm{A}$, p.Glu542Lys' was primarily predominant in the lung group. 'PIK3CA, c.3140A > G, p.His1047Arg' was primarily detected in the breast group. In one patient, 'PIK3CA, c.1624G $>\mathrm{A}$, p.Glu542Lys' was observed with $E G F R$ exon 19 deletion and T790M mutations. Nonsense mutations were infrequent in PIK3CA. PIK3CA signaling pathways serve a crucial role in replication, differentiation and apoptosis. EGFR activation promotes tumor growth, invasion and migration via the PIK3CA and mTOR pathways. PIK3CA mutations may cause resistance to $E G F R$-targeting therapies in patients with lung cancer $(20,21)$.

RET mutations were most frequent in the breast (8.8\%) and lung groups (4.8\%). The ' $R E T$, c.2324delinsGAC, p.Glu775Glyfs*6' mutation was most common and most of RET mutations were around the kinase domain (Fig. 4). $P T E N$ is one of the most commonly inactivated tumor suppressor genes in a wide variety of cancer types. PTEN loss and the activation of $\mathrm{PI} 3 \mathrm{~K} / \mathrm{mTOR}$ signaling are associated with EGFR TKIs resistance in patients with NSCLC $(20,21)$. PTEN mutations were observed in the breast $(4.4 \%)$ and lung $(6 \%)$ groups. 'PTEN, c. $397 \mathrm{G}>\mathrm{A}$, p.Val133Ile' was the most common mutation. Half of the PTEN mutations were nonsense or frameshift and most mutations were around the catalytic domain. Similarly, most $A P C$ mutations were nonsense or frameshift. While most germline $A P C$ mutations were missense, the majority of the somatic $A P C$ mutations were nonsense or frameshift. This may help to distinguish between germline and somatic mutations.

It has been reported that patients with EGFR exon 19 deletions who receive long-term EGFR-TKI therapy show a high prevalence of T790M mutations (22). In the present study, T790M mutation was detected in two patients who also exhibited the exon 19 deletion. In one patient, the 'ALK, c.3626delG, p.Arg1209Glnfs*49' mutation was detected and no ROS mutations were detected. Another patient exhibited an $A L K$ L1198F mutation in addition to the C1156Y mutation. L1198F substitution confers resistance to lorlatinib via steric interference with drug binding. However, L1198F paradoxically enhances binding to crizotinib, negating the effect of $\mathrm{C} 1156 \mathrm{Y}$ and re-sensitizing resistant cancer to crizotinib. The patient received crizotinib, following which cancer-associated symptoms and liver failure resolved (23). Further studies are needed to detect and clarify the effects of these neutralizing mutations.

Several studies have evaluated the concordance between $K R A S, B R A F$ and NRAS point mutations detected by ctDNA and tumor-tissue analysis $(24,25)$. More mutations were detected by ctDNA analysis than by tumor biopsy, reflecting the ability of liquid biopsy to reflect tumor heterogeneity. Moreover, the shorter turnaround time required to perform ctDNA analysis compared with tumor tissue analysis makes it possible to start treatment earlier (24). 

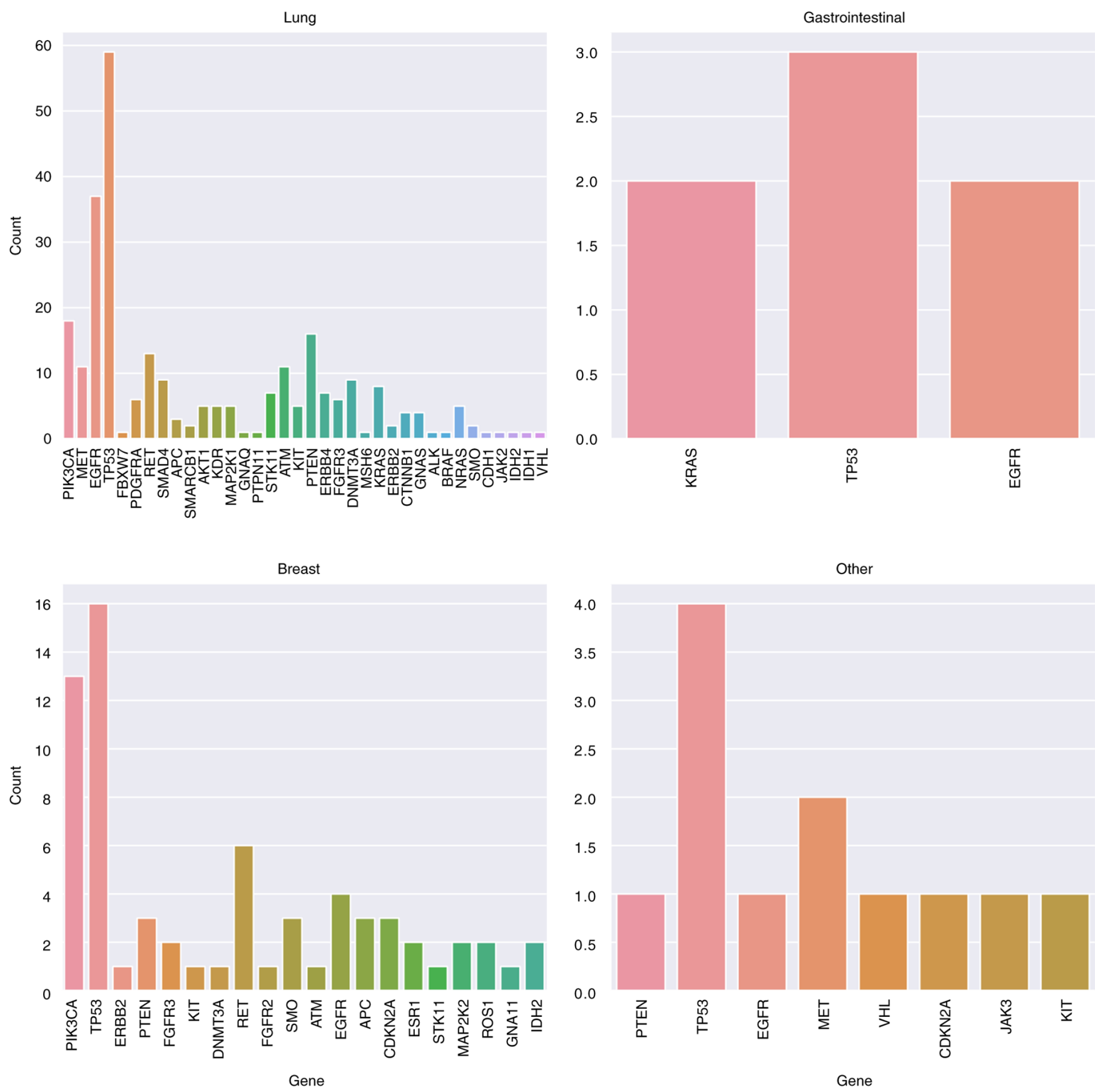

Figure 3. Most commonly mutated genes in the lung, breast, gastrointestinal and other groups.

Familial cancer syndromes account for $5-10 \%$ of all malignancies caused by inherited mutations; they raise the risk of tumor development and are typically characterized by early-onset cancer (26). In the present study, patients with high variant fraction mutations in APC, ATM, MLH1, MSH6, PTEN, PTPN11, RB1, RET, STK11, TP53, TSC1 and VHL underwent testing with a familial cancer panel. Even though the variant fractions of the mutations were between 1 and 10\%, there may be some risk associated with germline inheritance. Family screening and genetic counseling are important for suspected germline mutations, primarily when the variant fraction is between 50 and $100 \%$.

The majority of patients presented with advanced metastatic tumors from different cities around the country. Treatment and survival information could not be collected for all the patients and follow-up tests could not be performed. Only mutations that can be targeted with treatment were reported and discussed here. Despite these limitations, the liquid biopsy test helped to identify the resistance mechanism in most cases (Figs. 2, 3 and 4).

The treatment of patients with lung and breast cancer with PIK3CA, PTEN and RET mutations has not yet been defined. In the present study, PIK3CA mutations occurred in $\sim 6.6 \%$ of patients with lung adenocarcinoma and $19 \%$ of patients with breast cancer. It has been reported that PIK3CA-positive patients have a worse prognosis (2). PI3K/AKT/mTOR pathway inhibitors may be considered for patients with PIK3CA and PTEN mutations. To the best of our knowledge, the present 


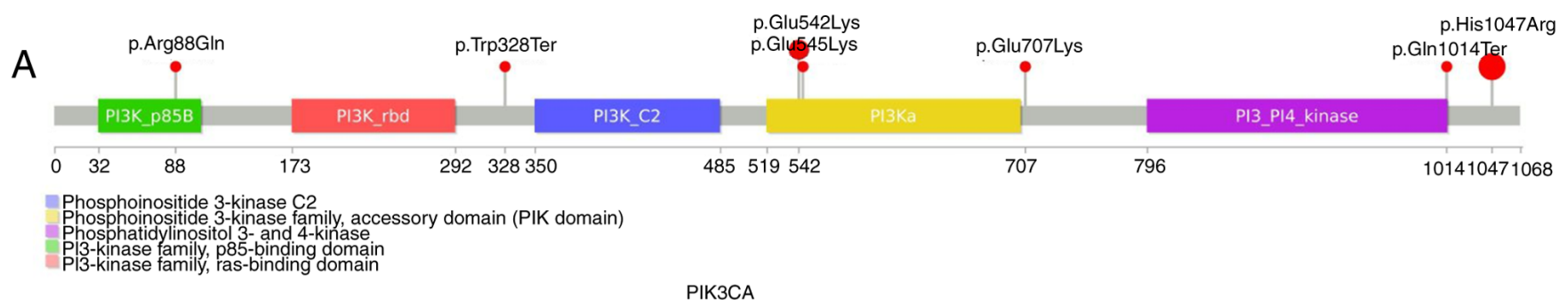

B

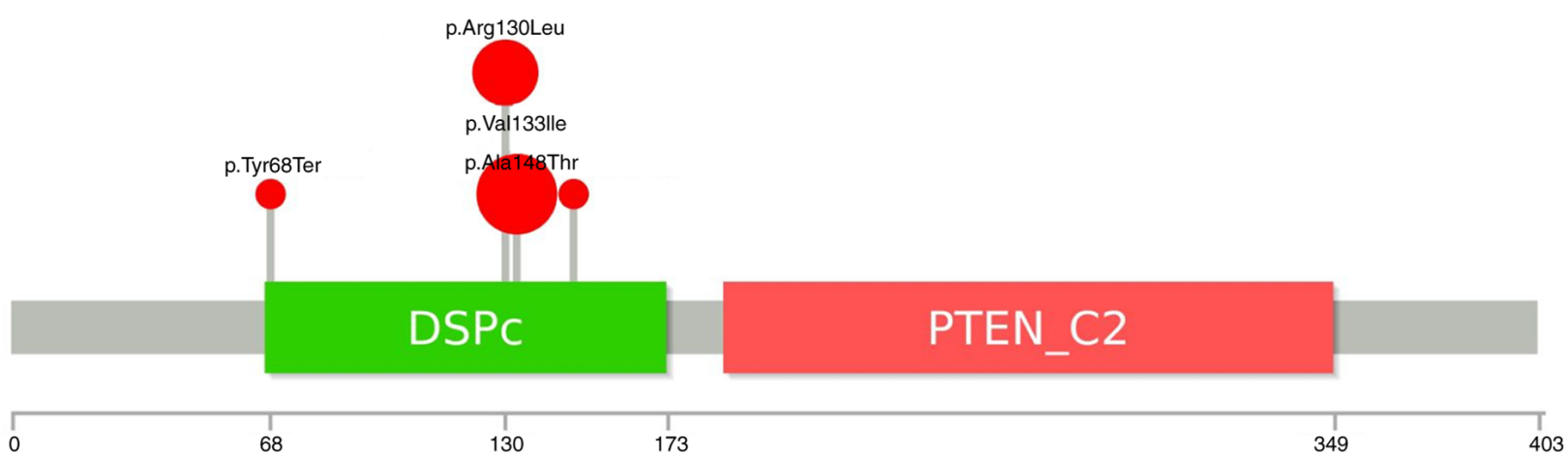

Dual specificity phosphatase, catalytic domain

C2 domain of PTEN tumour-suppressor protein

PTEN

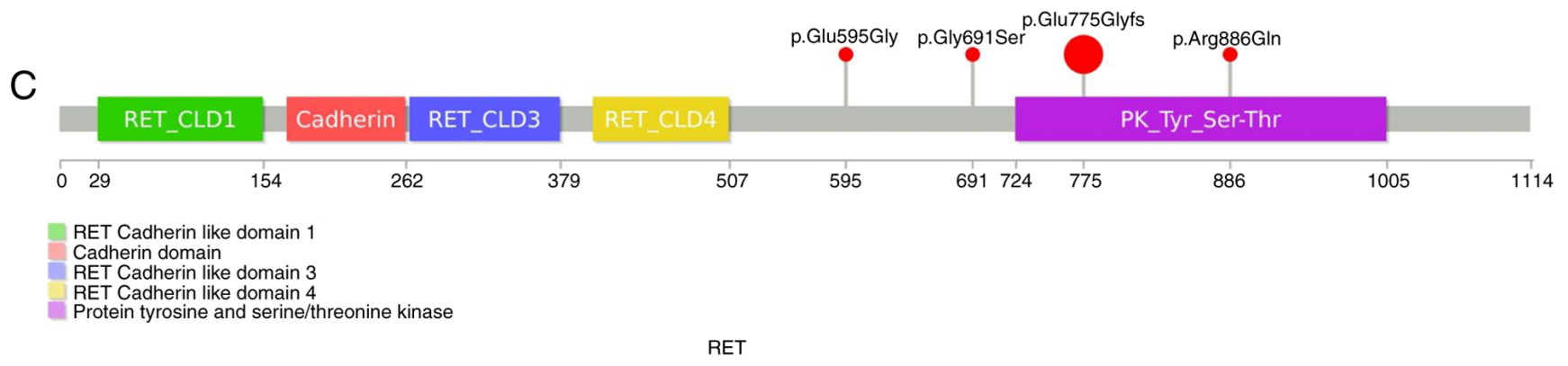

Figure 4. PIK3CA, PTEN, RET mutations detected. The most common mutations in (A) PIK3CA, (B) PTEN, (C) RET genes and associated domains.

study is the first to concentrate on PIK3CA, PTEN and RET mutations in the context of breast and lung adenocarcinoma and evaluate the genetic variability. The findings support the potential of using gene therapy to target mutant PIK3CA, PTEN and RET genes.

In conclusion, the findings of the present study suggested that patients with solid tumors, particularly lung and breast cancer, should undergo PIK3CA, PTEN and RET sequencing to assess clinical characteristics and prognosis. Greater understanding of the structure and mechanisms of $P I K 3 C A, P T E N$ and RET may help to inform more clinically meaningful therapeutic approaches for patients with cancer. Moreover, developments in assessing and researching novel variants of known cancer genes will serve a key role in improving individual cancer risk prediction, therapy and prognosis.

\section{Acknowledgements}

Not applicable.

\section{Funding}

No funding was received.

\section{Availability of data and materials}

The datasets generated and/or analyzed during the current study are not publicly available due to restrictions of the Ministry of Health of Turkey but are available from the corresponding author on reasonable request.

\section{Authors' contributions}

IS designed the study, analyzed and interpreted the results and wrote the manuscript. HS collected the data, analyzed and interpreted the results and reviewed the manuscript. SA and OD evaluated the patients and collected the clinical data. HBE and TB collected the data and analyzed and interpreted the results. All authors read and approved the final version of the manuscript. IS, HS, HBE and TB confirm the authenticity of all the raw data. 


\section{Ethics approval and consent to participate}

The present study was approved (approval no. 03/1072) by the Ethics Committee at the University of Health Sciences, Dr. Abdurrahman Yurtaslan Ankara Oncology Training and Research Hospital. Written informed consent was obtained from all patients.

\section{Patient consent for publication}

Not applicable.

\section{Competing interests}

The authors declare that they have no competing interests.

\section{Authors' information}

HS, ORCID no. 0000-0002-6087-5947; IS, ORCID no. 0000-0002-6050-816X.

\section{References}

1. Torre LA, Siegel RL, Ward EM and Jemal A: Global cancer incidence and mortality rates and trends-an update. Cancer Epidemiol Biomarkers Prev 25: 16-27, 2016.

2. Song Z, Yu X and Zhang Y: Mutation and prognostic analyses of $P I K 3 C A$ in patients with completely resected lung adenocarcinoma. Cancer Med 5: 2694-2700, 2016.

3. Mattiuzzi C, Sanchis-Gomar F and Lippi G: Concise update on colorectal cancer epidemiology. Ann Transl Med 7: 609, 2019.

4. Dafni U, Tsourti Z and Alatsathianos I: Breast cancer statistics in the european union: Incidence and survival across european countries. Breast Care 14: 344-353, 2019.

5. Franczak C, Filhine-Tressarieu P, Broséus J, Gilson P, Merlin JL and Harlé A: Clinical interest of circulating tumor DNA in oncology. Arch Med Res 49: 297-305, 2018.

6. Arneth B: Update on the types and usage of liquid biopsies in the clinical setting: A systematic review. BMC Cancer 18: 527, 2018.

7. Lindeman NI, Cagle PT, Aisner DL, Arcila ME, Beasley MB, Bernicker EH, Colasacco C, Dacic S, Hirsch FR, Kerr K, et al: Updated molecular testing guideline for the selection of lung cancer patients for treatment with targeted tyrosine kinase inhibitors: Guideline from the college of American pathologists, the international association for the study of lung cancer, and the association for molecular pathology. Arch Pathol Lab Med 142: 321-346, 2018.

8. Von Bubnoff N: Liquid biopsy: Approaches to dynamic genotyping in cancer. Oncol Res Treat 40: 409-416, 2017.

9. Bronte G, Ulivi P, Verlicchi A, Cravero P, Delmonte A and Crinò L: Targeting RET-rearranged non-small-cell lung cancer: Future prospects. Lung Cancer (Auckl) 10: 27-36, 2019.

10. Oxnard GR, Thress KS, Alden RS, Lawrance R, Paweletz CP, Cantarini M, Yang JC, Barrett JC and Jänne PA: Association between plasma genotyping and outcomes of treatment with osimertinib (AZD9291) in advanced non-small-cell lung cancer. J Clin Oncol 34: 3375-3382, 2016.

11. Liu S, Li S, Wang B, Liu W, Gagea M, Chen H, Sohn J, Parinyanitikul N, Primeau T, Do KA, et al: Cooperative effect of oncogenic MET and PIK3CA in an HGF-dominant environment in breast cancer. Mol Cancer Ther 18: 399-412, 2019.

12. Tan AC: Targeting the PI3K/Akt/mTOR pathway in non-small cell lung cancer (NSCLC). Thorac Cancer 11: 511-518, 2020.

13. Gkountakos A, Sartori G, Falcone I, Piro G, Ciuffreda L, Carbone C, Tortora G, Scarpa A, Bria E, Milella M, et al: PTEN in lung cancer: Dealing with the problem, building on new knowledge and turning the game around. Cancers (Basel) 11: $1141,2019$.
14. Jaiswal S, Fontanillas P, Flannick J, Manning A, Grauman PV, Mar BG, Lindsley RC, Mermel CH, Burtt N, Chavez A, et al: Age-related clonal hematopoiesis associated with adverse outcomes. N Engl J Med 371: 2488-2498, 2014.

15. Snyder MW, Kircher M, Hill AJ, Daza RM and Shendure J: Cell-free DNA comprises an in vivo nucleosome footprint that informs its tissues-of-origin. Cell 164: 57-68, 2016.

16. Gupta S, Provenzale D, Llor X, Halverson AL, Grady W, Chung DC, Haraldsdottir S, Markowitz AJ, Slavin TP Jr, Hampel H, et al: NCCN guidelines insights: Genetic/familial high-risk assessment: Colorectal, version 2.2019. J Natl Compr Cancer Netw 17: 1032-1041, 2019.

17. Daly MB, Pilarski R, Yurgelun MB, Berry MP, Buys SS, Dickson P, Domchek SM, Elkhanany A, Friedman S, Garber JE, et al: NCCN guidelines insights: Genetic/familial high-risk assessment: Breast, ovarian, and pancreatic, version 1.2020. J Natl Compr Cancer Netw 18: 380-391, 2020.

18. Li MM, Datto M, Duncavage EJ, Kulkarni S, Lindeman NI, Roy S, Tsimberidou AM, Vnencak-Jones CL, Wolff DJ, Younes A and Nikiforova MN: Standards and guidelines for the interpretation and reporting of sequence variants in cancer: A joint consensus recommendation of the association for molecular pathology, american society of clinical oncology, and college of american pathologists. J Mol Diagnostics 19: 4-23, 2017.

19. Jay JJ and Brouwer C: Lollipops in the clinic: Information dense mutation plots for precision medicine. PLoS One 11: e 0160519, 2016.

20. Guo Y, Song J, Wang Y, Huang L, Sun L, Zhao J, Zhang S, Jing W, Ma J and Han C: Concurrent genetic alterations and other biomarkers predict treatment efficacy of egfr-tkis in EGFR-mutant non-small cell lung cancer: A Review. Front Oncol 10: 610923, 2020.

21. Chang WJ, Sung JS, Lee SY, Kang EJ, Kwon NJ, Kim HM, Shin SW, Choi JY, Choi YJ, Kim JW, et al: The clinical significance of RAS, PIK3CA, and PTEN mutations in non-small cell lung cancer using cell-free DNA. J Clin Med 14: 2642, 2020.

22. Matsuo N, Azuma K, Sakai K, Hattori S, Kawahara A, Ishii H, Tokito T, Kinoshita T, Yamada K, Nishio K and Hoshino T: Association of EGFR Exon 19 deletion and EGFR-TKI treatment duration with frequency of T790M mutation in EGFR-mutant lung cancer patients. Sci Rep 6: 36458, 2016.

23. Shaw AT, Friboulet L, Leshchiner I, Gainor JF, Bergqvist S, Brooun A, Burke BJ, Deng YL, Liu W, Dardaei L, et al: Resensitization to crizotinib by the lorlatinib ALK resistance mutation L1198F . N Engl J Med 374: 54-61, 2016.

24. Thierry AR, El Messaoudi S, Mollevi C, Raoul JL, Guimbaud R, Pezet D, Artru P, Assenat E, Borg C, Mathonnet M, et al: Clinical utility of circulating DNA analysis for rapid detection of actionable mutations to select metastatic colorectal patients for anti-EGFR treatment. Ann Oncol 28: 2149-2159, 2017.

25. Formica V, Lucchetti J, Doldo E, Riondino S, Morelli C, Argirò R, Renzi N, Nitti D, Nardecchia A, Dell'Aquila E, et al: Clinical utility of plasma $K R A S, N R A S$ and $B R A F$ mutational analysis with real time PCR in metastatic colorectal cancer patients-the importance of tissue/plasma discordant cases. J Clin Med 10: 87, 2020.

26. Howlader N, Noone AM, Krapcho M, Garshell J, Miller D, Altekruse SF, Kosary CL, Yu M, Ruhl J, Tatalovich Z, Mariotto A, Lewis DR, Chen HS, Feuer EJ and Cronin KA (eds): SEER Cancer Statistics Review, 1975-2012, National Cancer Institute. Bethesda, MD, based on November 2014 SEER data submission, posted to the SEER web site, 2015. https://seer. cancer.gov/archive/csr/1975_2012. Accessed November 18, 2015.

This work is licensed under a Creative Commons Attribution-NonCommercial-NoDerivatives 4.0 International (CC BY-NC-ND 4.0) License. 